\title{
Focused Ion Beam Based Micromanipulation to Form Air Bridge Interconnect
}

\author{
C.L. Progl $1^{1}$, J.P. Vitarelli ${ }^{2}$, P.E. Russell ${ }^{1,2}$ \\ ${ }^{1}$ Analytical Instrumentation Facility and Materials Science and Engineering Dept., North \\ Carolina State University, Box 7531, Raleigh, NC, 27695 \\ ${ }^{2}$ Materials Analytical Services, Inc., 616 Hutton St., Suite 101, Raleigh, NC 27606 \\ In general Focused Ion Beam (FIB) systems are used for micromanipulation and sample \\ preparation for various microscopies. When the sample needs to be a live device as is the \\ case for Electron Beam Induced Current (EBIC) analysis problems are encountered \\ making electrical contact. FIB techniques for preparation of cross-sectional STEM-EBIC \\ samples have been developed [1]. However, these methods often entail several steps \\ which are time consuming, difficult and destructive to most of the device. In this paper a \\ novel sample preparation method for placing live electrical interconnects to a device is \\ presented.
}

In this particular example a light emitting diode (LED) is the device of interest and a functional cross-section sample is required. Figure 1.A. is a schematic of the proposed method and shows the live electrical interconnect which requires micromanipulation to place and attach. Typical FIB electrical interconnects are deposited across a surface, but this is not the case here as an air gap 100 microns must be spanned. The method starts with either a bare or extracted device (Fig. 1.B.) and from then all subsequent preparation steps are done in the FIB. The first step is a standard FIB lift-out from an area of interest. Note that depending on device size the first step will leave most of the die intact allowing for additional sampling from different areas. The extracted sample is then mounted to one side of a previously fabricated assembly consisting of two copper grids (contacts) electrically isolated from each other (Fig. 1.C.). The assembly serves as the sample stage and as the positive/negative contacts for EBIC analysis. The next step requires forming and manipulating into position the "positive" electrical interconnect between the sample and backside grid shown. A thinned tungsten wire ( $\sim 3$ micron diameter) was used in an initial attempt to make this contact, but the wire proved to be too rigid. Handling after FIB sample preparation caused the sample grid to flex and the tungsten wire interconnect failed. For the next attempt a test wafer with copper microtraces was treated in a buffered oxide etch to undercut and free the traces. The etched and cleaned test wafer was then platinum coated and placed in the FIB. The sampling probe was attached to a trace $(0.65$ micron wide) via FIB deposited Pt and slowly raised to extract the trace from the wafer (Fig. 2.A. - B.). The extracted trace was then attached with platinum to the second TEM contact (Fig. 2.C.). Static charge held the free end of the trace to the probe as it was spanned across the air gap and attached to the sample (Fig. 3.A.). Use of a copper trace provided the needed flexibility necessary for the sample to survive further handling (Fig. 3.B.) in addition to functioning as the positive electrical interconnect. This allows for SEM, STEM and EBIC analysis of the functional diode with controlled bias voltage applied. 


\section{References}

[1] K.L. Bunker et al., Electronic Device Failure Analysis, 4 (2002), 29
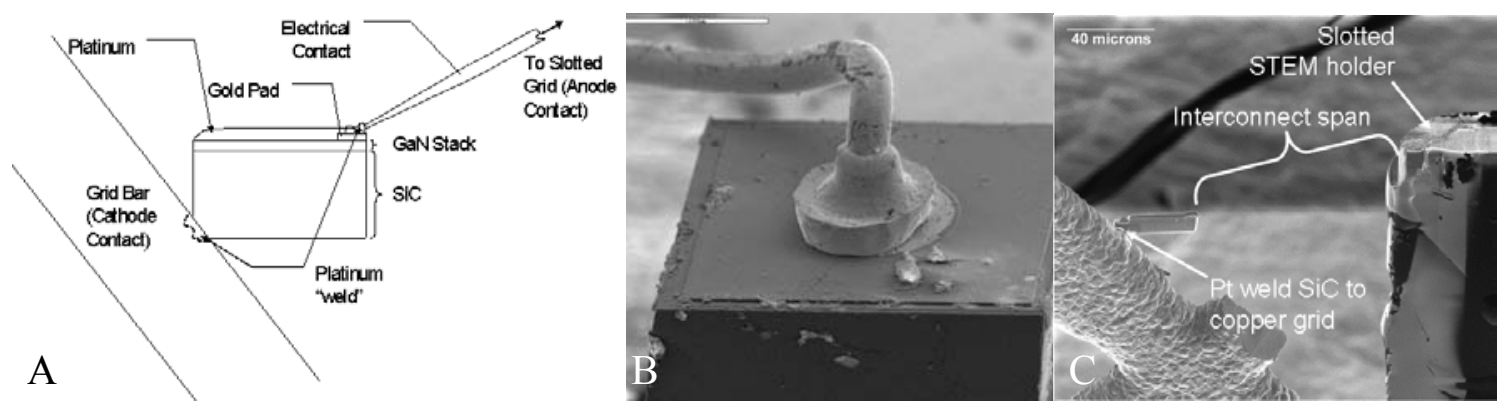

Fig. 1. A. Schematic of sample preparation concept. B. Extracted light emitting diode. C. Air gap between sample and grid that interconnect must span ( 100 microns).

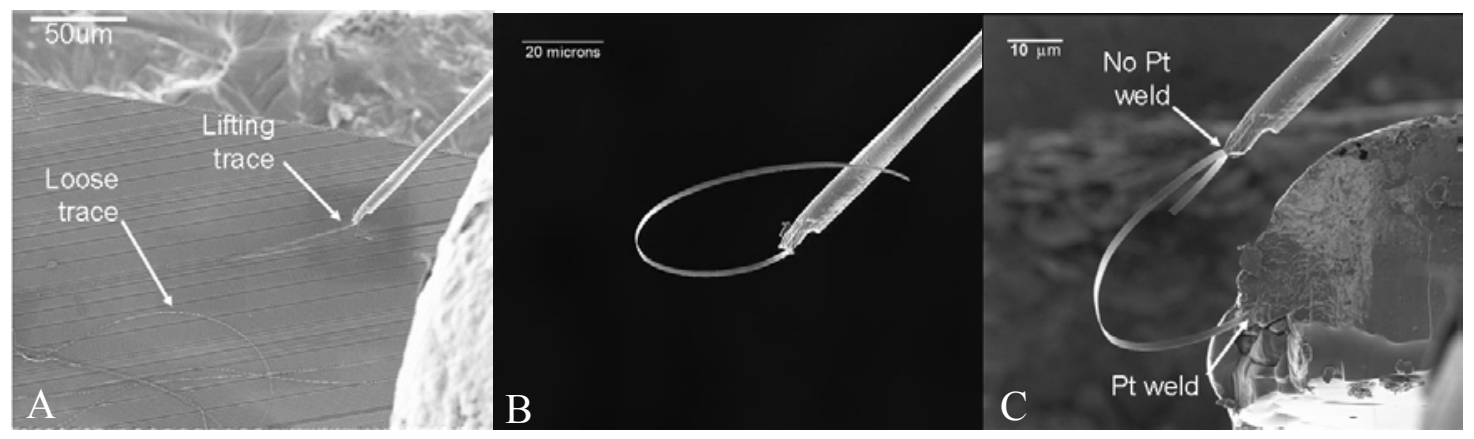

Fig. 2. A. Lift-off of 0.65 wide micron copper trace from test wafer. B. Extracted copper trace. C. One end of trace attached to grid and free end held by static charge ready to span air gap.

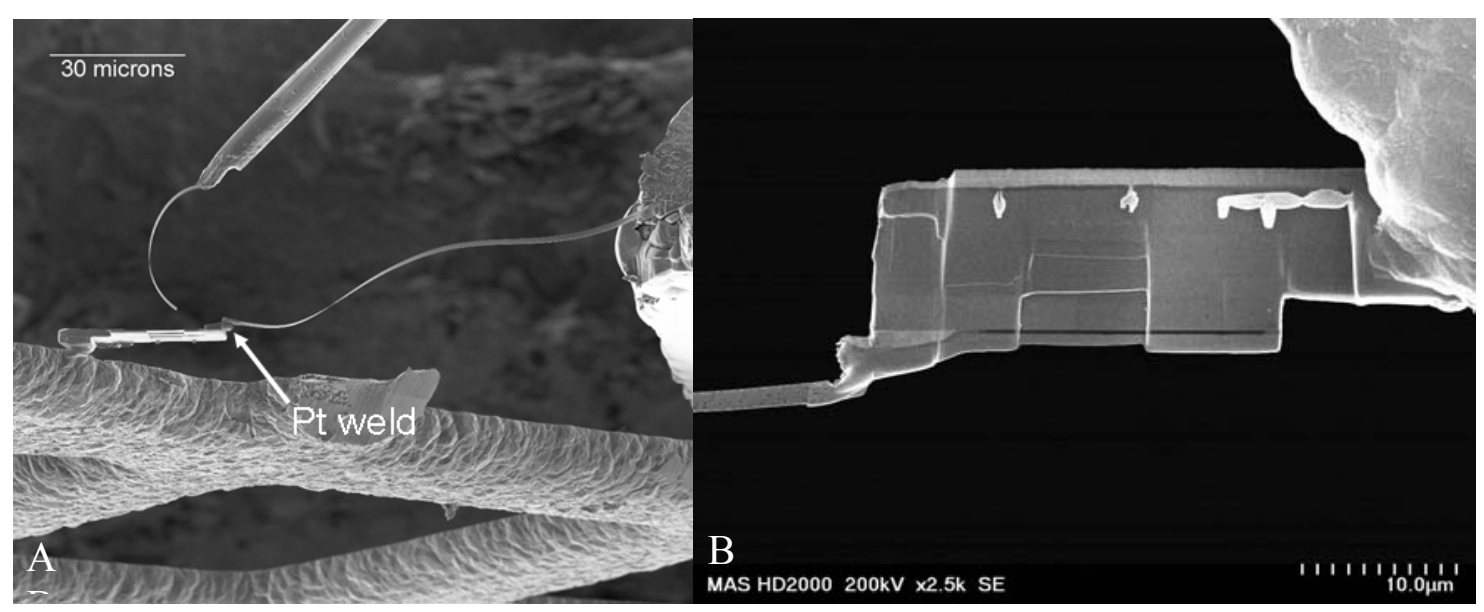

Fig. 3. A. Completed electrical interconnect. B. STEM image of finished sample. 